Supporting information

\title{
Design and interfacial assembly of a new series of gemini amphiphiles with hydrophilic poly(ethyleneamine) spacers
}

Tifeng Jiao, Guocheng Zhang, and Minghua Liu*

Beijing National Laboratory for Molecular Science (BNLMS), CAS Key Laboratory of Colloid and Interface Science, Institute of Chemistry, CAS, Beijing 100080, P. R. China.

\section{Basic Data for the compounds}

$N, N$ '-Bis(4-octadecyloxy-salicylidene)-diethylenetriamine (GN1): MS (MALDI-TOF, m/z): $849.0\left(\mathrm{C}_{54} \mathrm{H}_{93} \mathrm{~N}_{3} \mathrm{O}_{4}\right.$, cal. 848.35). ${ }^{1} \mathrm{H}$ NMR $\left(\mathrm{CDCl}_{3}, \delta \mathrm{ppm}\right): 0.88\left(\mathrm{~m}, \mathrm{CH}_{3}, 6 \mathrm{H}\right)$, 1.26-1.66 (m, $\left.\mathrm{CH}_{2}, 60 \mathrm{H}\right), 1.78\left(\mathrm{~m}, \mathrm{CH}_{2}, 4 \mathrm{H}\right), 2.98\left(\mathrm{t}, \mathrm{CH}_{2}, 4 \mathrm{H}\right), 3.66\left(\mathrm{t}, \mathrm{CH}_{2}, 4 \mathrm{H}\right), 3.96$ (t, $\left.\mathrm{CH}_{2} \mathrm{O}, 4 \mathrm{H}\right), 6.35(\mathrm{~m}, 4 \mathrm{H}),, 7.07(\mathrm{~d}, 2 \mathrm{H}, \mathrm{J}=8.4 \mathrm{~Hz}), 8.18(\mathrm{~s}, \mathrm{HC}=\mathrm{N}, 2 \mathrm{H})$, and 14.72 (s, OH, 2H). Anal. Calcd. for $\mathrm{C}_{54} \mathrm{H}_{93} \mathrm{~N}_{3} \mathrm{O}_{4}: \mathrm{C}, 76.45 \% ; \mathrm{H}, 11.05 \%$;, $4.95 \%$. Found: $\mathrm{C}$, $76.56 \% ; \mathrm{H}, 11.32 \% ; \mathrm{N}, 4.82 \%$.

$N, N$ '-Bis(4-octadecyloxy-salicylidene)-triethylenetetramine $\quad$ (GN2): $\quad$ MS (MALDI-TOF, m/z): $891.5\left(\mathrm{C}_{56} \mathrm{H}_{98} \mathrm{~N}_{4} \mathrm{O}_{4}\right.$, cal. 891.42). ${ }^{1} \mathrm{H}$ NMR $\left(\mathrm{CDCl}_{3}, \delta \mathrm{ppm}\right): 0.88$ (m, $\left.\mathrm{CH}_{3}, 6 \mathrm{H}\right), 1.26-1.66$ (m, $\left.\mathrm{CH}_{2}, 60 \mathrm{H}\right), 1.77$ (m, $\left.\mathrm{CH}_{2}, 4 \mathrm{H}\right), 2.75\left(\mathrm{t}, \mathrm{CH}_{2}, 8 \mathrm{H}\right), 3.64$ (t, $\left.\mathrm{CH}_{2}, 4 \mathrm{H}\right), 3.95\left(\mathrm{t}, \mathrm{CH}_{2} \mathrm{O}, 4 \mathrm{H}\right), 6.35(\mathrm{~m}, 4 \mathrm{H}), 7.10(\mathrm{~m}, 2 \mathrm{H}), 8.10(\mathrm{~s}, \mathrm{HC}=\mathrm{N}, 2 \mathrm{H})$, and 14.32 (s, OH, 2H). Anal. Calcd. for $\mathrm{C}_{56} \mathrm{H}_{98} \mathrm{~N}_{4} \mathrm{O}_{4}: \mathrm{C}, 75.45 \% ; \mathrm{H}, 11.08 \%$; N, $6.29 \%$. Found: C, 75.56\%; H, 11.32\%; N, 6.42\%. 
$N, N^{\prime}$-Bis(4-octadecyloxy-salicylidene)-tetraethylene pentamine (GN3): $\quad$ MS (MALDI-TOF, m/z): $934.2\left(\mathrm{C}_{58} \mathrm{H}_{103} \mathrm{~N}_{5} \mathrm{O}_{4}\right.$, cal. 934.49). ${ }^{1} \mathrm{H}$ NMR $\left(\mathrm{CDCl}_{3}, \delta \mathrm{ppm}\right): 0.88$ (m, $\left.\mathrm{CH}_{3}, 6 \mathrm{H}\right), 1.26-1.66\left(\mathrm{~m}, \mathrm{CH}_{2}, 60 \mathrm{H}\right), 1.77\left(\mathrm{~m}, \mathrm{CH}_{2}, 4 \mathrm{H}\right), 2.68\left(\mathrm{t}, \mathrm{CH}_{2}, 12 \mathrm{H}\right), 3.55$ (t, $\left.\mathrm{CH}_{2}, 4 \mathrm{H}\right), 3.96\left(\mathrm{t}, \mathrm{CH}_{2} \mathrm{O}, 4 \mathrm{H}\right), 6.28(\mathrm{~m}, 4 \mathrm{H}), 7.42(\mathrm{~m}, 2 \mathrm{H}), 8.09(\mathrm{~s}, \mathrm{HC}=\mathrm{N}, 2 \mathrm{H})$, and 14.12 (s, OH, 2H). Anal. Calcd. for $\mathrm{C}_{58} \mathrm{H}_{103} \mathrm{~N}_{5} \mathrm{O}_{4}$ : C, 74.55\%; H, 11.11\%; N, $7.49 \%$. Found: C, 74.65\%; H, 11.22\%; N, 7.63\%. 